Commun. Korean Math. Soc. 25 (2010), No. 1, pp. 1-9

DOI 10.4134/CKMS.2010.25.1.001

\title{
ON PERMUTING 3-DERIVATIONS AND COMMUTATIVITY IN PRIME NEAR-RINGS
}

\author{
Kyoo-Hong Park And Yong-SoO Jung
}

\begin{abstract}
In this note, we introduce a permuting 3-derivation in nearrings and investigate the conditions for a near-ring to be a commutative ring.
\end{abstract}

\section{Introduction and preliminaries}

A non-empty set $R$ with two binary operations + (addition) and - (multiplication) is called a near-ring if it satisfies the following axioms:

i) $(R,+)$ is a group (not necessarily abelian),

ii) $(R, \cdot)$ is a semigroup,

iii) $x \cdot(y+z)=x \cdot y+x \cdot z$ for all $x, y, z \in R$.

Exactly speaking, it is a left near-ring because it satisfies the left distributive law. We will use the word near-ring to mean left near-ring and denote $x y$ instead of $x \cdot y$.

For a near-ring $R$, the set $R_{0}=\{x \in R: 0 x=0\}$ is called the zerosymmetric part of $R$. A near-ring $R$ is said to be zero-symmetric if $R=R_{0}$. Throughout this note, $R$ will be a zero-symmetric near-ring and $R$ is called prime if $x R y=\{0\}$ implies $x=0$ or $y=0$. Recall that $R$ is called $n$ torsion-free, where $n$ is a positive integer, if $n x=0$ implies $x=0$ for all $x \in R$. The symbol $C$ will represent the multiplicative center of $R$, that is, $C=\{x \in R: x y=y x$ for all $y \in R\}$. For $x \in R$, the symbol $C(x)$ will denote the centralizer of $x$ in $R$. As usual, for $x, y \in R,[x, y]$ will denote the commutator $x y-y x$, while $\langle x, y\rangle$ will indicate the additive-group commutator $x+y-x-y$. As for terminologies concerning near-rings used here without special mention, we refer to G. Pilz [6].

An additive map $d: R \rightarrow R$ is called a derivation if the Leibniz rule $d(x y)=$ $d(x) y+x d(y)$ holds for all $x, y \in R$. By a bi-derivation we mean a bi-additive map $D: R \times R \rightarrow R$ (i.e., $D$ is additive in both arguments) which satisfies the

Received November 9, 2008; Revised December 13, 2008.

2000 Mathematics Subject Classification. 16W20, 16Y30, 16 Y99.

Key words and phrases. prime near-ring, commutative ring, permuting 3-derivation. 
relations

$$
D(x y, z)=D(x, z) y+x D(y, z)
$$

and

$$
D(x, y z)=D(x, y) z+y D(x, z)
$$

for all $x, y, z \in R$. Let $D$ be symmetric, that is, $D(x, y)=D(y, x)$ for all $x, y \in R$. The map $\tau: R \rightarrow R$ defined by $\tau(x)=D(x, x)$ for all $x \in R$ is called the trace of $D$.

A map $F: R \times R \times R \rightarrow R$ is said to be permuting if the equation $F\left(x_{1}, x_{2}, x_{3}\right)=F\left(x_{\pi(1)}, x_{\pi(2)}, x_{\pi(3)}\right)$ holds for all $x_{1}, x_{2}, x_{3} \in R$ and for every permutation $\{\pi(1), \pi(2), \pi(3)\}$. A map $f: R \rightarrow R$ defined by $f(x)=F(x, x, x)$ for all $x \in R$, where $F: R \times R \times R \rightarrow R$ is a permuting map, is called the trace of $F$. It is obvious that, in the case $F: R \times R \times R \rightarrow R$ is a permuting map which is also 3 -additive (i.e., additive in each argument), the trace $f$ of $F$ satisfies the relation

$$
f(x+y)=f(x)+2 F(x, x, y)+F(x, y, y)+F(x, x, y)+2 F(x, y, y)+f(y)
$$

for all $x, y \in R$.

Since we have

$$
F(0, y, z)=F(0+0, y, z)=F(0, y, z)+F(0, y, z)
$$

for all $y, z \in R$, we obtain $F(0, y, z)=0$ for all $y, z \in R$. Hence we get

$$
0=F(0, y, z)=F(x-x, y, z)=F(x, y, z)+F(-x, y, z)
$$

and so we see that $F(-x, y, z)=-F(x, y, z)$ for all $x, y, z \in R$. This tells us that $f$ is an odd function.

A 3-additive map $\Delta: R \times R \times R \rightarrow R$ will be called a 3 -derivation if the relations

and

$$
\begin{gathered}
\Delta\left(x_{1} x_{2}, y, z\right)=\Delta\left(x_{1}, y, z\right) x_{2}+x_{1} \Delta\left(x_{2}, y, z\right), \\
\Delta\left(x, y_{1} y_{2}, z\right)=\Delta\left(x, y_{1}, z\right) y_{2}+y_{1} \Delta\left(x, y_{2}, z\right)
\end{gathered}
$$

$$
\Delta\left(x, y, z_{1} z_{2}\right)=\Delta\left(x, y, z_{1}\right) z_{2}+z_{1} \Delta\left(x, y, z_{2}\right)
$$

are fulfilled for all $x, y, z, x_{i}, y_{i}, z_{i} \in R, i=1,2$.

For example, let $N$ be a noncommutative near-ring and let

$$
R=\left\{\left(\begin{array}{ll}
a & b \\
0 & 0
\end{array}\right) \mid a, b \in N\right\} .
$$

It is clear that $R$ is a noncommutative near-ring under matrix addition and matrix multiplication. We define a map $\Delta: R \times R \times R \rightarrow R$ by

$$
\left(\left(\begin{array}{cc}
a_{1} & b_{1} \\
0 & 0
\end{array}\right),\left(\begin{array}{cc}
a_{2} & b_{2} \\
0 & 0
\end{array}\right),\left(\begin{array}{cc}
a_{3} & b_{3} \\
0 & 0
\end{array}\right)\right) \mapsto\left(\begin{array}{cc}
0 & a_{1} a_{2} a_{3} \\
0 & 0
\end{array}\right) .
$$

Then it is easy to see that $\Delta$ is a 3 -derivation.

Derivations and bi-derivations in rings and near-rings have been studied by many mathematicians in several ways $[1,2,3,4,5,7,9,10]$. Furthermore, 
M. Uçkun and M. A. Öztürk [8] investigated symmetric bi- $\Gamma$-derivations and commutativity in $\Gamma$-near-rings.

In this note, we examine the conditions for a near-ring with permuting 3derivations to be a commutative ring.

\section{Lemmas}

We need the following lemmas to obtain our main results in Section 3.

Lemma 2.1 ([2, Lemma 3]). Let $R$ be a prime near-ring. If $C \backslash\{0\}$ contains an element $z$ for which $z+z \in C$, then $(R,+)$ is abelian.

Lemma 2.2. Let $R$ be a 3!-torsion free near-ring. Suppose that there exists a permuting 3-additive map $F: R \times R \times R \rightarrow R$ such that $f(x)=0$ for all $x \in R$, where $f$ is the trace of $F$. Then we have $F=0$.

Proof. For any $x, y \in R$,

$$
f(x+y)=f(x)+2 F(x, x, y)+F(x, y, y)+F(x, x, y)+2 F(x, y, y)+f(y)
$$

and so, by the hypothesis, we get

$$
2 F(x, x, y)+F(x, y, y)+F(x, x, y)+2 F(x, y, y)=0
$$

for all $x, y \in R$. Putting $-x$ instead of $x$ in (2.1), we obtain

$$
2 F(x, x, y)-F(x, y, y)+F(x, x, y)-2 F(x, y, y)=0
$$

for all $x, y \in R$.

On the other hand, for any $x, y \in R$,

$$
f(y+x)=f(y)+2 F(y, y, x)+F(y, x, x)+F(y, y, x)+2 F(y, x, x)+f(x)
$$

and thus, by the hypothesis, we have

$$
2 F(x, y, y)+F(x, x, y)+F(x, y, y)+2 F(x, x, y)=0
$$

for all $x, y \in R$ since $F$ is permuting. Comparing (2.1) with (2.2), we get

$$
2 F(x, y, y)+F(x, x, y)+F(x, y, y)=F(x, x, y)-3 F(x, y, y)
$$

which implies that

$$
\begin{aligned}
& 2 F(x, y, y)+F(x, x, y)+F(x, y, y)+2 F(x, x, y) \\
= & F(x, x, y)-3 F(x, y, y)+2 F(x, x, y)
\end{aligned}
$$

for all $x, y \in R$. Hence it follows from (2.3) that

$$
F(x, x, y)-3 F(x, y, y)+2 F(x, x, y)=0
$$

for all $x, y \in R$. The substitution $x=-x$ in (2.4) leads to

$$
F(x, x, y)+3 F(x, y, y)+2 F(x, x, y)=0
$$

for all $x, y \in R$. Combining (2.4) and (2.5), we obtain

$$
F(x, y, y)=0
$$


for all $x, y \in R$ since $R$ is 6 -torsion free. The replacement $y=y+z$ to linearize (2.6) yields

$$
F(x, y, z)=0
$$

for all $x, y, z \in R$, i.e., $F=0$ which completes the proof.

Lemma 2.3. Let $R$ be a 3!-torsion free prime near-ring and let $x \in R$. Suppose that there exists a nonzero permuting 3-derivation $\Delta: R \times R \times R \rightarrow R$ such that $x \delta(y)=0$ for all $y \in R$, where $\delta$ is the trace of $\Delta$. Then we have $x=0$.

Proof. Since we have

$$
\delta(y+z)=\delta(y)+2 \Delta(y, y, z)+\Delta(y, z, z)+\Delta(y, y, z)+2 \Delta(y, z, z)+\delta(z)
$$

for all $y, z \in R$, the hypothesis gives

$$
2 x \Delta(y, y, z)+x \Delta(y, z, z)+x \Delta(y, y, z)+2 x \Delta(y, z, z)=0
$$

for all $y, z \in R$. Setting $y=-y$ in (2.7), it follows that

$$
2 x \Delta(y, y, z)-x \Delta(y, z, z)+x \Delta(y, y, z)-2 x \Delta(y, z, z)=0
$$

for all $y, z \in R$.

On the other hand, for any $y, z \in R$,

$$
\delta(z+y)=\delta(z)+2 \Delta(z, z, y)+\Delta(z, y, y)+\Delta(z, z, y)+2 \Delta(z, y, y)+\delta(y)
$$

and so, by the hypothesis, we have

$$
2 x \Delta(y, z, z)+x \Delta(y, y, z)+x \Delta(y, z, z)+2 x \Delta(y, y, z)=0
$$

for all $x, y, z \in R$ since $\Delta$ is permuting. Comparing (2.7) with (2.8), we get

$$
2 x \Delta(y, z, z)+x \Delta(y, y, z)+x \Delta(y, z, z)=x \Delta(y, y, z)-3 x \Delta(y, z, z)
$$

which means that

$$
\begin{aligned}
& 2 x \Delta(y, z, z)+x \Delta(y, y, z)+x \Delta(y, z, z)+2 x \Delta(y, y, z) \\
= & x \Delta(y, y, z)-3 x \Delta(y, z, z)+2 x \Delta(y, y, z)
\end{aligned}
$$

for all $x, y, z \in R$. Now, from (2.9), we obtain

$$
x \Delta(y, y, z)-3 x \Delta(y, z, z)+2 x \Delta(y, y, z)=0
$$

for all $x, y, z \in R$. Taking $y=-y$ in (2.10) leads to

$$
x \Delta(y, y, z)+3 x \Delta(y, z, z)+2 x \Delta(y, y, z)=0
$$

for all $x, y, z \in R$. Combining (2.10) and (2.11), we obtain

$$
x \Delta(y, z, z)=0
$$

for all $x, y \in R$ since $R$ is 6 -torsion free. Replacing $z=z+w$ to linearize (2.12) and using the conditions show that

$$
x \Delta(w, y, z)=0
$$

for all $w, x, y, z \in R$. Substituting $w v$ for $w$ in (2.13), we get

$$
x w \Delta(v, y, z)=0
$$


for all $v, w, x, y, z \in R$. Since $R$ is prime and $\Delta \neq 0$, we arrive at $x=0$. This completes the proof of the theorem.

Lemma 2.4. Let $R$ be a near-ring and let $\Delta: R \times R \times R \rightarrow R$ be a permuting 3-derivation. Then we have

$$
[\Delta(x, z, w) y+x \Delta(y, z, w)] v=\Delta(x, z, w) y v+x \Delta(y, z, w) v
$$

for all $v, w, x, y, z \in R$.

Proof. Since we have

$$
\Delta(x y, z, w)=\Delta(x, z, w) y+x \Delta(y, z, w)
$$

for all $w, x, y, z \in R$, the associative law gives

$$
\begin{aligned}
\Delta((x y) v, z, w) & =\Delta(x y, z, w) v+x y \Delta(v, z, w) \\
& =[\Delta(x, z, w) y+x \Delta(y, z, w)] v+x y \Delta(v, z, w)
\end{aligned}
$$

for all $v, w, x, y, z \in R$ and

$$
\begin{aligned}
\Delta(x(y v), z, w) & =\Delta(x, z, w) y v+x \Delta(y v, z, w) \\
& =\Delta(x, z, w) y v+x[\Delta(y, z, w) v+y \Delta(v, z, w)] \\
& =\Delta(x, z, w) y v+x \Delta(y, z, w) v+x y \Delta(v, z, w)
\end{aligned}
$$

for all $v, w, x, y, z \in R$. Comparing (2.14) and (2.15), we see that

$$
[\Delta(x, z, w) y+x \Delta(y, z, w)] v=\Delta(x, z, w) y v+x \Delta(y, z, w) v
$$

for all $v, w, x, y, z \in R$. The proof of the lemma is complete.

\section{Permuting 3-derivations and commutativity}

Now we are ready to prove our main results in this section.

Theorem 3.1. Let $R$ be a 3!-torsion free prime near-ring. Suppose that there exists a nonzero permuting 3 -derivation $\Delta: R \times R \times R \rightarrow R$ such that

$$
\Delta(x, y, z) \in C
$$

for all $x, y, z \in R$. Then $R$ is a commutative ring.

Proof. Assume that $\Delta(x, y, z) \in C$ for all $x, y, z \in R$. Since $\Delta$ is nonzero, there exist $x_{0}, y_{0}, z_{0} \in R$ such that $\Delta\left(x_{0}, y_{0}, z_{0}\right) \in C \backslash\{0\}$ and

$$
\Delta\left(x_{0}, y_{0}, z_{0}\right)+\Delta\left(x_{0}, y_{0}, z_{0}\right)=\Delta\left(x_{0}, y_{0}, z_{0}+z_{0}\right) \in C .
$$

So $(R,+)$ is abelian by Lemma 2.1 .

Since the hypothesis implies that

$$
w \Delta(x, y, z)=\Delta(x, y, z) w
$$

for all $w, x, y, z \in R$, we replace $x$ by $x v$ in (3.1) to get

$$
w[\Delta(x, y, z) v+x \Delta(v, y, z)]=[\Delta(x, y, z) v+x \Delta(v, y, z)] w
$$


and thus, from Lemma 2.4 and the hypothesis, it follows that

$$
\Delta(x, y, z) w v+\Delta(v, y, z) w x=\Delta(x, y, z) v w+\Delta(v, y, z) x w
$$

which means that

$$
\Delta(x, y, z)[w, v]=\Delta(v, y, z)[x, w]
$$

for all $v, w, x, y, z \in R$. Setting $\delta(u)$ in place of $v$ in (3.2) and using $\delta(x) \in C$ for all $x \in R$ by the hypothesis, we obtain

$$
\Delta(\delta(u), y, z)[x, w]=0
$$

for all $u, w, x, y, z \in R$. The substitution $v x$ for $x$ in (3.3) yields that

$$
\Delta(\delta(u), y, z) v[x, w]=0
$$

for all $u, v, w, x, y, z \in R$. Since $R$ is prime, we obtain either $\Delta(\delta(u), y, z)=0$ or $[x, w]=0$ for all $u, w, x, y, z \in R$.

Assume that

$$
\Delta(\delta(u), y, z)=0
$$

for all $u, y, z \in R$. Let us take $u+x$ instead of $u$ in (3.4). Then we obtain

$$
\begin{aligned}
0 & =\Delta(\delta(u+x), y, z) \\
& =\Delta(\delta(u)+\delta(x)+3 \Delta(u, u, x)+3 \Delta(u, x, x), y, z) \\
& =3 \Delta(\Delta(u, u, x), y, z)+3 \Delta(\Delta(u, x, x), y, z),
\end{aligned}
$$

that is,

$$
\Delta(\Delta(u, u, x), y, z)+\Delta(\Delta(u, x, x), y, z)=0
$$

for all $v, w, x, y \in R$. Setting $u=-u$ in (3.5) and then comparing the result with (3.11), we see that

$$
\Delta(\Delta(u, u, x), y, z)=0
$$

for all $u, x, y, z \in R$. Substituting $u x$ for $x$ in (3.6) and employing (3.4) give the relation

$$
\delta(u) \Delta(x, y, z)+\Delta(u, y, z) \Delta(u, u, x)=0
$$

and so it follows from the hypothesis that

$$
\delta(u) \Delta(x, y, z)+\Delta(u, u, x) \Delta(u, y, z)=0
$$

for all $u, x, y, z \in R$. We put $u=y=x$ in (3.7) to obtain

$$
\delta(x) \Delta(x, x, w)=0
$$

for all $w, x \in R$. Taking $w x$ in substitute for $w$ in (3.8) yields

$$
\delta(x) w \delta(x)=0
$$

and so the primeness of $R$ implies that $\delta(x)=0$ for all $x \in R$. Hence, by Lemma 2.2 , we have $\Delta=0$ which is a contradiction. So $R$ is a commutative ring. This proves the theorem. 
Theorem 3.2. Let $R$ be a 3!-torsion free prime near-ring. Suppose that there exists a nonzero permuting 3-derivation $\Delta: R \times R \times R \rightarrow R$ such that

$$
\delta(x), \delta(x)+\delta(x) \in C(\Delta(u, v, w))
$$

for all $u, v, w, x \in R$, where $\delta$ is the trace of $\Delta$. Then $R$ is a commutative ring.

Proof. Assume that

$$
\delta(x), \delta(x)+\delta(x) \in C(\Delta(u, v, w))
$$

for all $u, v, w, x \in R$. From (3.9), we get

$$
\begin{aligned}
& \Delta(u+t, v, w)(\delta(x)+\delta(x)) \\
= & (\delta(x)+\delta(x)) \Delta(u+t, v, w) \\
= & (\delta(x)+\delta(x))[\Delta(u, v, w)+\Delta(t, v, w)] \\
= & (\delta(x)+\delta(x)) \Delta(u, v, w)+(\delta(x)+\delta(x)) \Delta(t, v, w) \\
= & \delta(x) \Delta(u, v, w)+\delta(x) \Delta(u, v, w)+\delta(x) \Delta(t, v, w)+\delta(x) \Delta(t, v, w) \\
= & \delta(x)[\Delta(u, v, w)+\Delta(u, v, w)+\Delta(t, v, w)+\Delta(t, v, w)] \\
= & {[\Delta(u, v, w)+\Delta(u, v, w)+\Delta(t, v, w)+\Delta(t, v, w)] \delta(x) }
\end{aligned}
$$

for all $t, u, v, w, x \in R$ and

$$
\begin{aligned}
& \Delta(u+t, v, w)(\delta(x)+\delta(x)) \\
= & \Delta(u+t, v, w) \delta(x)+\Delta(u+t, v, w) \delta(x) \\
= & {[\Delta(u, v, w)+\Delta(t, v, w)] \delta(x)+[\Delta(u, v, w)+\Delta(t, v, w)] \delta(x) } \\
= & {[\Delta(u, v, w)+\Delta(t, v, w)+\Delta(u, v, w)+\Delta(t, v, w)] \delta(x) }
\end{aligned}
$$

for all $t, u, v, w, x \in R$. Comparing (3.10) and (3.11), we obtain

$$
\Delta(\langle u, t\rangle, v, w) \delta(x)=0
$$

for all $t, u, v, w, x \in R$. Hence it follows from Lemma 2.3 that

$$
\Delta(\langle u, t\rangle, v, w)=0
$$

for all $t, u, v, w \in R$. We substitute $u z$ for $u$ and $u t$ for $t$ in (3.12) to get

$$
\begin{aligned}
0 & =\Delta(u\langle z, t\rangle, v, w) \\
& =\Delta(u, v, w)\langle z, t\rangle+u \Delta(\langle z, t\rangle, v, w) \\
& =\Delta(u, v, w)\langle z, t\rangle .
\end{aligned}
$$

That is,

$$
\Delta(u, v, w)\langle z, t\rangle=0
$$

for all $t, u, v, w, z \in R$. Letting $z=s z$ and $t=s t$ in (3.13) yields

$$
\Delta(u, v, w) s\langle z, t\rangle=0
$$


for all $s, t, u, v, w, z \in R$. Since $\Delta \neq 0$, we conclude, from (3.14) and the primeness of $R$, that $\langle z, t\rangle=0$ is fulfilled for all $t, z \in R$. Therefore $(R,+)$ is abelian.

By the hypothesis, we know that

$$
[\delta(x), \Delta(u, v, w)]=0
$$

for all $u, v, w, x \in R$. Hence if we let $x=x+y$ in (3.15), then we deduce from (3.15) that

$$
[\Delta(x, x, y), \Delta(u, v, w)]+[\Delta(x, y, y), \Delta(u, v, w)]=0
$$

for all $u, v, w, x, y \in R$. Setting $y=-y$ in (3.16) and comparing the result with (3.16), we obtain

$$
[\Delta(x, y, y), \Delta(u, v, w)]=0
$$

for all $u, v, w, x, y \in R$. Replacing $y$ by $y+z$ in (3.17) and using (3.17), we have

$$
[\Delta(x, y, z), \Delta(u, v, w)]=0
$$

since $\Delta$ is permuting, i.e.,

$$
\Delta(x, y, z) \Delta(u, v, w)=\Delta(u, v, w) \Delta(x, y, z)
$$

for all $u, v, w, x, y, z \in R$. Taking $u t$ instead of $u$ in (3.18), we obtain

$$
\begin{aligned}
& \Delta(u, v, w) t \Delta(x, y, z)-\Delta(x, y, z) \Delta(u, v, w) t \\
& \quad+u \Delta(t, v, w) \Delta(x, y, z)-\Delta(x, y, z) u \Delta(t, v, w)=0
\end{aligned}
$$

for all $t, u, v, w, x, y, z \in R$. Substituting $\delta(u)$ for $u$ in (3.19) and then utilizing the hypothesis and (3.18), we get

$$
\Delta(\delta(u), v, w)[t, \Delta(x, y, z)]=0
$$

for all $t, u, v, w, x, y, z \in R$. Let us write in (3.20) ws instead of $w$. Then we have

$$
\Delta(\delta(u), v, w) s[t, \Delta(x, y, z)]=0
$$

for all $s, t, u, v, w, x, y, z \in R$. Since $R$ is prime, we arrive at either $\Delta(\delta(u), v, w)$ $=0$ or $[t, \Delta(x, y, z)]=0$ for all $t, u, v, w, x, y, z \in R$.

As in the proof of Theorem 3.1, the case when $\Delta(\delta(u), v, w)=0$ holds for all $u, v, w \in R$ leads to the contradiction.

Consequently, we arrive at

$$
[t, \Delta(x, y, z)]=0
$$

for all $t, x, y, z \in R$, i.e, $\Delta(x, y, z) \in C$ for all $x, y, z \in R$. Therefore, Theorem 3.1 yields that $R$ is a commutative ring which is complete the proof. 


\section{References}

[1] M. Ashraf, A. Ali, and S. Ali, $(\sigma, \tau)$-derivations on prime near-rings, Archivum Mathematicum (BRNO), Tomus. 40 (2004), 281-286.

[2] H. E. Bell and G. Mason, On derivations in near-rings, in Near-Rings and Near-Fields (Tübingen, 1985), G. Betsch, Ed., vol. 137 of North-Holland Mathematics Studies, pp. 31-35, North-Holland, Amsterdam, The Netherlands, 1987.

[3] M. Brešar, Commuting maps: a survey, Taiwanese J. Math. 8 (2004), no. 3, 361-397.

[4] Y. Çeven and M. A. Öztürk, Some properties of symmetric bi- $(\sigma, \tau)$-derivations in near-rings, Commun. Korean Math. Soc. 22 (2007), no. 4, 487-491.

[5] Y.-S. Jung and K.-H. Park, On prime and semiprime rings with permuting 3derivations, Bull. Korean Math. Soc. 44 (2007), no. 4, 789-794.

[6] G. Pilz, Near-rings, 2nd Ed. North Holland, Amsterdam, 1983.

[7] E. C. Posner, Derivations in prime rings, Proc. Amer. Math. Soc. 8 (1957), 10931100.

[8] M. Uçkun and M. A. Öztürk, On trace of symmetric bi-gamma-derivations in gammanear-rings, Houston J. Math. 33 (2007), no. 2, 323-339.

[9] J. Vukman, Symmetric bi-derivations on prime and semi-prime rings, Aequationes Math. 38 (1989), 245-254.

[10] - Two results concerning symmetric bi-derivations on prime rings, Aequationes Math. 40 (1990), 181-189.

KYOO-HONG PARK

Department of Mathematics Education

SeOWon University

Cheonguu 361-742, Korea

E-mail address: parkkh@seowon.ac.kr

YONG-SOO JUNG

Department of Mathematics

Sun MoON UNIVERSITY

ASAN 336-708, KoREA

E-mail address: ysjung@sunmoon.ac.kr 